\title{
Detecting System Designed for Express Industry
}

\author{
Haoda Gong ${ }^{1, a^{*}}$, Xingzhe Jiang ${ }^{2, b}$, Xiaoyu Ye ${ }^{2, c}$ \\ ${ }^{1}$ No.2 High School Affiliated to East China Normal University, China \\ ${ }^{2}$ High School Affiliated to Shanghai Jiao Tong University in Jiading, China \\ ${ }^{3} U M-S J T U$ Joint Institute, China \\ agxh1998@126.com, bjordan313@126.com cjamesgign@hotmaill.com
}

\begin{abstract}
Keywords: Detecting System, Smart Express, Embedded System, Bluetooth, Integrated Sensors Abstract. Chinese express industry lacks regulation. Once the parcel is broken during the express journey, it is difficult to determine who should be responsible for the loss because there are lots of steps in express. Hence, our solution is to design a machine that can record the damage the parcel suffering and send a message to the customer, which is our smart express. Our product uses sensors to detect damage and use GPS to record the time and location. When the parcel is damaged, it sends these information to customers. To complete our project, we need to learn how to use the STM32 developing board, make the pierce detection, write a program and buildup a prototype.
\end{abstract}

\section{Introduction}

The express industry has been developing in high speed. However, there is a serious problem in the express industry. Chinese express industry lacks regulation, and the safety of the goods is hard to be guaranteed, especially for some precious and fragile commercials. The common phenomenon is that the worker of express company process the package rudely to save time, which damages the insides goods even the consignee do not notice that. Once the parcel is broken during the express journey, it is difficult to determine who should be responsible for the compensation because there are lots of steps in express[1].

Moreover, there is no system to share the instantaneous information of the package with the customers and their parcel. It is impossible for the costumer to know the treatment of their package immediately. Although express service develops fast, today there are still some serious problems:

Difficult to get the feedback of instantaneous status of the package

Unknown treatment to the package in delivery

No standard and professional qualification for express company

\section{Solution-Smart Express}

A new smart express system has accurate detectors and convenient feedback system which provides: Accurate crash and pierce detectors

\section{Instantaneous GPS coordinates}

\section{An feedback system with massage and website function}

Based on the needs mentioned before, the "Smart Express" is designed to fit the situation. The accelerometer, pierce detector and GPS are combined to an STM32 board to collect the data of the product. The GSM/GPRS module is also attached to the control board in order to send the information to the customers. The website is set up in the Windows Azure sever for customer feedback.

The procedure of the system is better illustrated by Figure 1. The system is enclosed in the parcels to detect the accidents to the products. The accelerometer is used for crash detection by measuring the instantaneous acceleration from time to time. A defaulted value is getting by simulative experiments and programmed in STM32 chip. When an object is crashed or hit, which as a result is measured as a peak value by the accelerometer, a warning message is sent to the user's cell phone. The GPS coordinate is recorded simultaneously, and the data is asynchronized to the cloud sever via GPRS 
signal. Likewise, the pierce detection part uses the enameled wire to detect whether the parcel has been pierced or not. The same operation is executed when the current signal tells the piercing action.

The feedback system consists of two parts: SMS warning and a website. User would receive a warning message if the parcel is crashed or pierced and visit smartexpress.chinacloudapp.cn to see more detailed information of the parcel, including accidents, location and time.

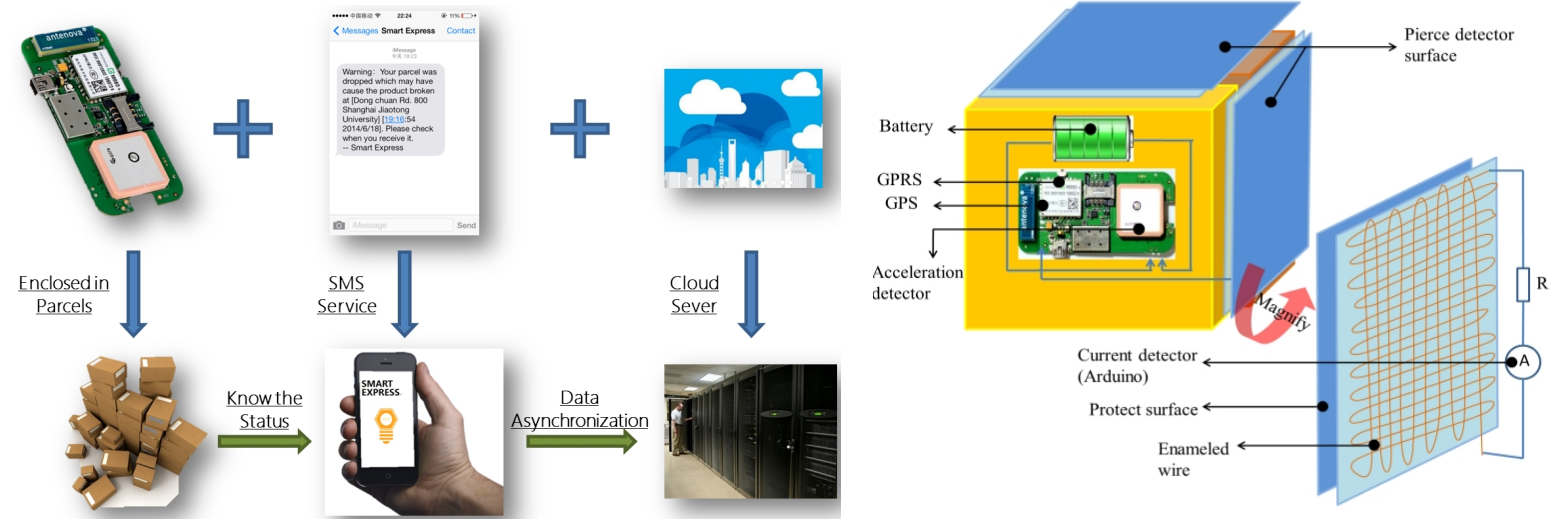

Figure 1\&2. Logic Chart and Concept Diagram of the "Smart Express"

Figure 2 is the major components of the system. All the electronical components is put inside a foam with $13 \mathrm{~cm} * 5 \mathrm{~cm} * 2 \mathrm{~cm}$ in size, which can be put into express boxes of average size. The system is enclosed in the parcels.It consists of three parts: the STM32 board, the Arduino board, and the enameled wire. The STM32 board integrates four individual modules: the GPS, the GPRS, theGSM, and the accelerometer. The accelerometer is used for crash detection; the enamaled wire and Arduino board is used for pierce detection; the GPS module will get the geographical coordinate when the parcel is crashed or pierced.

\section{Study the feature of GPS module}

The GPS module is integrated on the STM32 developing board which can be used to get the geographical coordinates by instructions.Figure 3 shows the SBAS Principle the GPS module uses to locate the position[2]. SBAS (Satellite Based Augmentation System) is an augmentation technology for GPS, which calculates GPS integrity and correction data with RIMS (Ranging and Integrity Monitoring Stations) on the ground and uses geostationary satellites (GEOs) to broadcast GPS integrity and correction data to GPS users. The correction data is transmitted on the GPS L1 frequency (1575.42 MHz), and therefore no additional receiver is required tomake use of the correction- and integrity data[3].

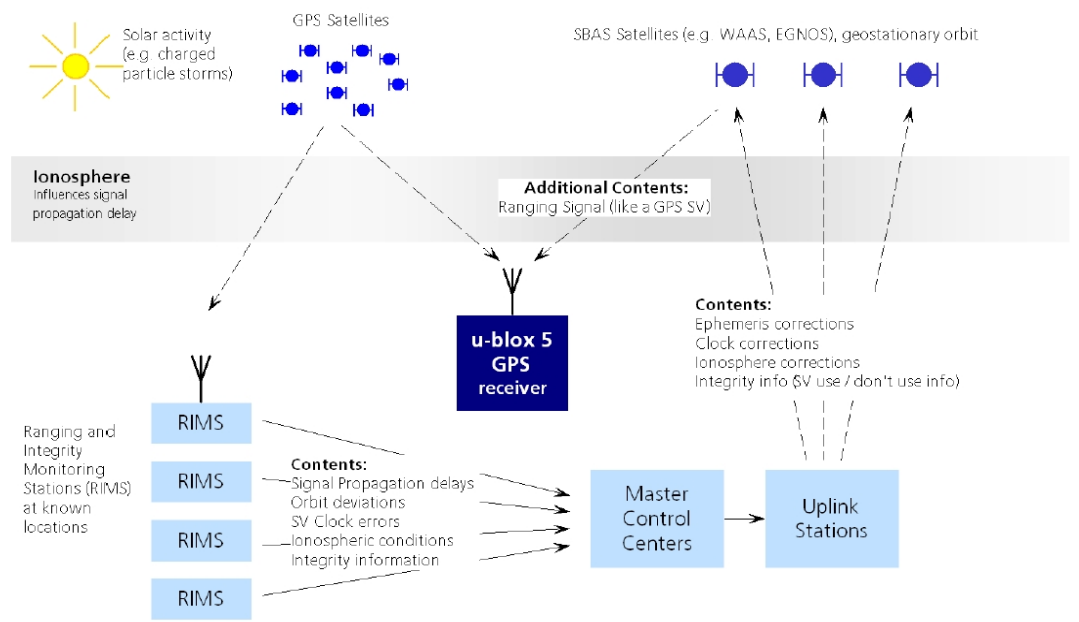

Figure 3. The SBAS Principle 


\section{Study the feature of the STM32 Developing Board}

The STM32 chip is the microprogrammed control unit (MUC) on the board. The controllling and processing programms of the GPS, GPRS, GSM modules are written on the MCU to get the coordinates, sent out data, and send short message, respectively.

The chip for data processing is STM32F103x6. The STM32F103xx performance line family incorporates the high-performance ARMCortex-M3 32-bit RISC core operating at a $72 \mathrm{MHz}$ frequency, high-speed embeddedmemories, and an extensiverange of enhanced I/Os and peripherals connected to two APB buses[4]. All devices offer two12-bit ADCs, three general purpose 16-bit timers plus one PWM timer, as well as standardand advanced communication interfaces. Figure 4. shows the schematical circuit if the chip.

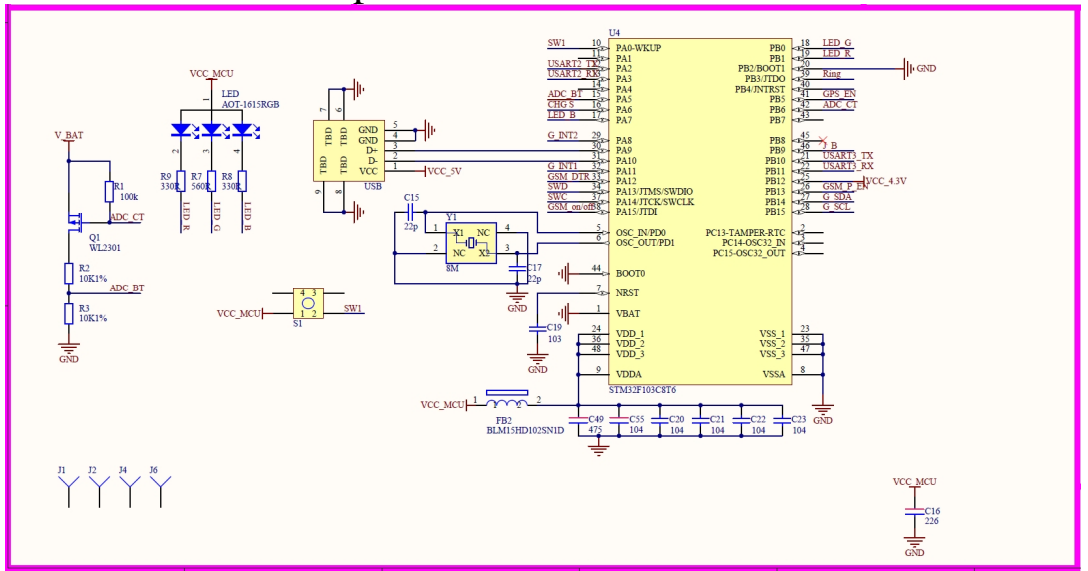

Figure 4. The Schematic of the STM32F103x6 Chip

\section{Crash Detection}

The acceleration is measured by the acclerometer respect to time. The flow chart shows the detection of crash accidents. When the acceleration becomes much larger in a small time interval, and decreases sharply after the time interval, the impulse is a large value in such circumstance[5]. The GPS coordinate is recorded and a warning message is sent to the user. Figure 5 shows the flow chart of the program logic of the crash detection[6].

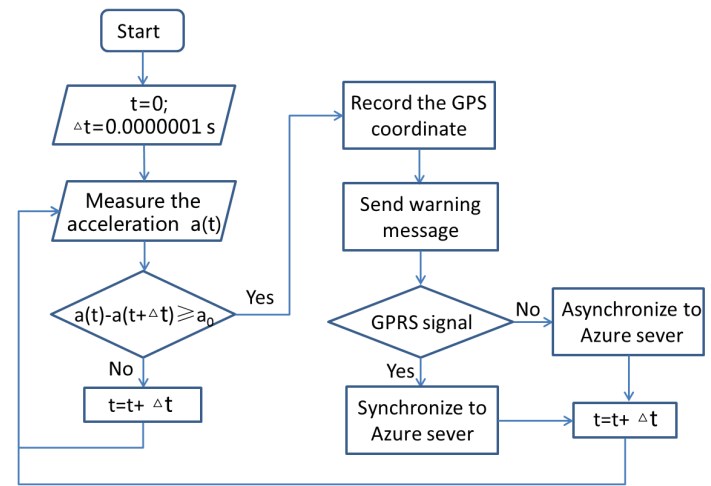

Figure 5. Flow Chart of the Program Logic of the Crash Detection

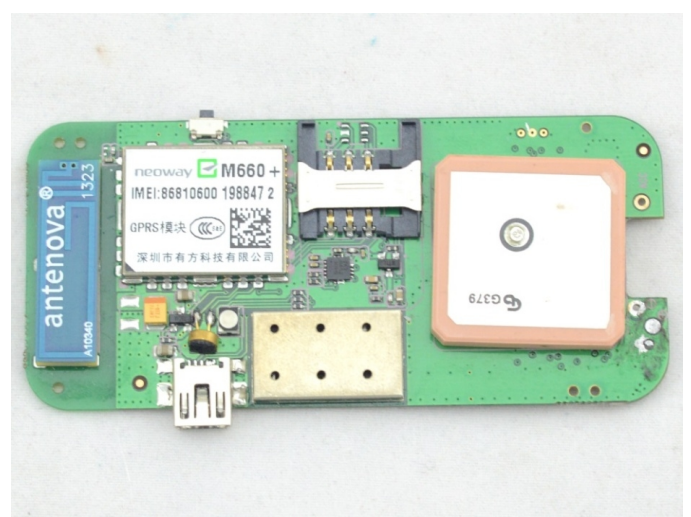

Figure 6.Shows the Integrated Modules Responsible for Crash Detection

\section{Test of GPS Module}

In order to know where the GPS module can receive and send signals as expectation, we tested the GPS module at different places to see whether it can report the location of itself. The results are as follows: 


\begin{tabular}{|l|l|l|}
\hline Place & GPS Module Succeeded & Connection speed \\
\hline On the streets & Yes & Fast \\
\hline Library & No & - \\
\hline Beside corridor windows in dorm & Yes & Fast \\
\hline Inside small cars & Yes & Slow \\
\hline Between two close buildings & No & - \\
\hline Student canteen & No & - \\
\hline Hall of Dongxia Building & Yes & Medium \\
\hline Dormitory rooms & No & - \\
\hline
\end{tabular}

Table 1: Test Results on GPS Module

From this test we can see that GPS works well in open areas, normally in semi-open area like small cars or halls of buildings. It does not work in closed space as the signal is blocked. However, we just expect it to work only at some critical spots to describe the travel route of the package, therefore it fulfills our needs.

\section{Test of GSM Module}

GSM needs to send short messages to specified cell phone number every time it is told to. Hence, we use sample driving program from the official site of the GSM chip to send short messages to our group members respectively. Here is the result:

\begin{tabular}{|l|l|l|l|}
\hline Phone number & Owner & Times of sending message & Rate of Successful sending \\
\hline $151 X X X X 7510$ & Chen Zhengxu & 10 & $100 \%$ \\
\hline $150 X X X X 5981$ & Li Chenhao & 15 & $100 \%$ \\
\hline $159 X X X X 3397$ & Ye Xiaoyu & 16 & $93.75 \%$ \\
\hline $150 X X X X 8707$ & Jiang Lu & 3 & $100 \%$ \\
\hline
\end{tabular}

Table 2: Test Results on Message Sending Function

From the table, we realised that we can send messages successfully almost every time unless some unexpected accident happens, this makes us system robust and reliable.

\section{Test of GPRS Module}

We will also need to upload some collected data to network, so GPRS module should find signal base station and send data to them quickly. We did some simple tests to measure the speed of transferring information via GPRS chip at different places:

\begin{tabular}{|l|l|}
\hline Place & GPRS uploading speed \\
\hline On the streets & $7.1 \mathrm{~KB} / \mathrm{s}$ \\
\hline Library & $13.2 \mathrm{~KB} / \mathrm{s}$ \\
\hline Beside corridor windows in dorm & $3.0 \mathrm{~KB} / \mathrm{s}$ \\
\hline Inside small cars & $8.4 \mathrm{~KB} / \mathrm{s}$ \\
\hline Between two close buildings & $10.2 \mathrm{~KB} / \mathrm{s}$ \\
\hline Student canteen & $6.7 \mathrm{~KB} / \mathrm{s}$ \\
\hline Hall of Dongxia Building & $20.5 \mathrm{~KB} / \mathrm{s}$ \\
\hline Dormitory rooms & $4.8 \mathrm{~KB} / \mathrm{s}$ \\
\hline
\end{tabular}

Table 3: Uploading Speed of GPRS Module at Different Places 
Note that we will approximately transfer 50 bytes data every time, even at the worst situation $(3 \mathrm{~KB} / \mathrm{s})$, we can upload 60 sets of data to the remote server in one second, which is far more than we need.

\section{Website building}

The website is inspired by some well known search engines such as google and baidu, we put just our logo, slogan and an input box on the website[7]. Users need to input the waybill numbers of their parcels, hence according to http protocol, we wrap this input box into a post form so that the remote server can receive the information in that input box.

\section{Smart Express}

Give us the number, we give the answer!

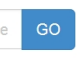

Figure 7: Homepage of "Smart Express"

After receiving the input waybill number, the server will look it up in our database, then it will get a list recording events happening on the package. Then, the server will send back all the retrieved data wrapped in a new web page back to the client side. The web page looks like the following picture:

On the left side of the page, we have a button which will redirecting customers back to the main searching page, a table listing all the events about the parcel and their time. At the bottom, we provide a progress bar to tell users the current progress of their delivery. On the right side of the page, we draw the travel route of the package and label all the places where the package gets hit or pierced.

The information in the left table is just a kind of reshaping our processed data, while the map on right requires to plug in Baidu Map Application Programming Interface and convert all the location data to the route on the map. Fortunately, Baidu corporation allows developers to use their tools like this freely, so we have nothing to worry about.

\section{Conclusion}

Smart Express aims at detecting the accidents to the parcels sent by the express company as well as regulating the industry. The system consists of four parts: crash detection, pierce detection, GPS locating, and feedback system.

The accelerometer is used for crash detection by measuring the instantaneous acceleration from time to time. The GPS coordinate will be recorded simultaneously and the data will be asynchronized to the cloud sever via GPRS signal. Likewise, the pierce detection part uses the enameled wire to detect whether the parcel has been pierced or not. The same operation will be executed when the current signal tells the piercing action.

The feedback system consists of two parts: SMS warning and a website. User will receive a warning message if the parcel is crashed or pierced and visit smartexpress.chinacloudapp.cn to see more detailed information of the parcel, including accidents, location and time.

Smart Express is new in the accident detecting of the parcels. The sensors and modules are highly integrated and low power consumption. The pierce detection part is a new method as well as a low cost way to detect whether the parcel has been pierced.

During the process of building up the system, we have learned such skills as writing embedded programs in STM32 chip, set up website on Windows Azure cloud sever, and designing simple circuit as a sensor to solve the problem. 
The system performed well in both simulative and real life tests. It is a reliable system that can be applied in the market place. It will be a revolution to the whole express industry if any express company would like to use it in their customer service.

\section{References}

[1]TechWeb.com. (2013, Mar. 13). Express industry[Online]:Available:

http://www.techweb.com.cn/data/2014-03-13/2015884.shtml

[2] N. Bulusu , GPS-less low-cost outdoor localization for very small devices , Personal

Communications, IEEE, Oct 2000 , pages 28-34.

[3] E.D.Kaplan, Understanding GPS: Principles and Applications , ARTECH HOUSE , 685

Canton Street, 2006 , Chapters 4-5.

Chapter 2 and Chapter 3.

[4]Atasheet5 Company, Performance line, ARM-based 32-bit MCU with Flash, USB, CAN,seven 16-bit timers, two ADCs and nine communication interfaces.

[5] K. Okada ,Detector for force and acceleration using resistance element , Nov 1990,

Chapter 1 and Chapter 2.

[6] S. Kabizaki, Mounting arrangement for acceleration detector element , May 1997 ,

[7]u-blox 5NMEA, UBX Protocol Specification, <www.u-blox.com>. 